\begin{tabular}{|c|c|c|c|}
\hline $\begin{array}{c}\text { Case Reports in } \\
\text { Cicitis }\end{array}$ & \begin{tabular}{|l|} 
Case Rep Oncol 2010;3:391-396 \\
DOI: 10.1159/000322135
\end{tabular} & \begin{tabular}{|l} 
Published online: \\
November 6, 2010
\end{tabular} & $\begin{array}{l}\text { ( ) } 2010 \text { S. Karger AG, Basel } \\
\text { ISSN } 1662-6575 \\
\text { www.karger.com/cro }\end{array}$ \\
\hline
\end{tabular}

\title{
Complete Metabolic Response with Recanalization of Portal Vein Tumor Thrombosis after Sunitinib in a Patient with Advanced Hepatocellular Carcinoma
}

\author{
Michele Basso $^{\mathrm{a}} \quad$ Maria Basso $^{\mathrm{b}} \quad$ Alessandro laculli $^{\mathrm{a}}$ \\ Maurizio Pompilib Laura Riccardi ${ }^{b}$ Brunella Barbaroc \\ Vittoria Rufini ${ }^{d}$ Alessandra Cassano $^{a}$ Paola Castaldi ${ }^{d}$ \\ Carlo Barone ${ }^{a}$
}

Divisions of a Medical Oncology and ${ }^{b}$ Hepatology, Department of Internal Medicine; 'Department of Radiology and ${ }^{\mathrm{d}}$ Institute of Nuclear Medicine, Catholic University of the Sacred Heart, Rome, Italy

\section{Key Words}

Hepatocellular carcinoma - Metabolic disease - Portal vein tumor thrombosis - Sorafenib . Sunitinib

\begin{abstract}
The prognosis of patients with advanced hepatocellular carcinoma $(\mathrm{HCC})$ is very poor. The outcome of these patients is particularly bleak when the disease is complicated by portal vein tumor thrombosis (PVTT), since the increased portal pressure often causes serious gastrointestinal bleedings. Before the introduction of sorafenib (SOR), a tyrosine kinase inhibitor, no effective treatment was available for patients with advanced disease. SOR is now considered the standard treatment even for patients with tumor thrombosis, although the well-known interference between tyrosine kinase inhibitors and the coagulation pathway calls for caution against their use in this setting. Here, we report the case of a 74-year-old male patient with advanced HCC and PVTT treated with sunitinib (SUN), another multikinase inhibitor. During the third cycle, our patient experienced a life-threatening hematemesis with hemorrhagic shock that required intensive care treatment and SUN discontinuation. However, he completely recovered, and the PET/CT scan performed 1 year after the adverse effect demonstrated no evidence of the tumor together with portal vein recanalization. The short course of SUN causing both tumor response and gastrointestinal bleeding warrants further studies on the
\end{abstract}


effectiveness of SUN in this setting as well as on the duration of treatment with multikinase inhibitors in patients with tumor thrombosis.

\section{Introduction}

Portal vein tumor thrombosis (PVTT) is one of the worst prognostic factors in patients with hepatocellular carcinoma (HCC) originating from liver cirrhosis. It can be observed in about $30 \%$ of HCC patients $[1,2]$, and the average survival of these patients after the diagnosis is very poor (2.7-4.0 months) [3]. Death is often due either to a progressive increase in portal pressure, resulting in gastrointestinal bleeding, or to the decrease in portal vein flow leading to ascites, jaundice, encephalopathy and liver failure. Until a few months ago, best supportive care was considered a reasonable choice for patients with such a critical prognosis, since transarterial chemoembolization entails a high risk of ischemic damage [4] and chemotherapy was largely ineffective. Some authors reported high survival rates in patients treated with a combination of transarterial chemoembolization and surgery with thrombectomy [5], but this procedure may be used only in highly selected patients.

Since sorafenib (SOR) has been shown to improve survival in patients with advanced HCC [6], this drug has become the standard treatment even for patients whose disease was complicated by PVTT. So far, however, there is no clear evidence concerning the effect of SOR in this subset of patients and their outcome. On the other hand, the wellknown interference between tyrosine kinase inhibitors and the coagulation pathway [7] calls for caution against their use in this setting.

Here, we describe a case of multifocal HCC with PVTT showing an unexpected favorable outcome after a short course of sunitinib (SUN), a tyrosine kinase inhibitor which is currently under investigation for use in the treatment of intermediate-advanced HCC.

\section{Case Report}

A 72-year-old male patient affected by chronic HCV-related hepatitis underwent percutaneous alcohol injection for a nodule of the sixth segment of the liver. Two years later, an abdominal CT scan demonstrated a massive thrombus involving the main portal vein and its branches, with infiltrative (not evaluable according to RECIST) neoplastic pattern of the sixth segment (fig. 1a 1 -a3). Histologic and cytologic examination of a needle biopsy of the infiltrative lesion and a fine needle aspiration of the portal vein thrombus demonstrated a poorly differentiated trabecular HCC with tumor thrombosis. Child-Pugh score was A6 with MELD score 10, and the tumor stage was BCLC-C. Serum albumin, ammonium, creatinine and coagulation parameters were normal; blood chemistry documented only a slight increase in transaminases (ALT 66 IU/l and AST 57 IU/l), alkaline phosphatase (377 IU/l) and $\gamma$ glutamyltransferase (122 IU/l). Blood count showed mild anemia (hemoglobin $11.2 \mathrm{~g} / \mathrm{dl}$ ) and thrombocytopenia $\left(84 \times 10^{3} / \mathrm{mm}^{3}\right)$. There was no ascites, and serum $\alpha$-fetoprotein was $3 \mathrm{ng} / \mathrm{ml}$. No distant metastases were detected on a whole-body CT scan and bone scintigraphy, and upper endoscopy showed no esophageal varices. ${ }^{18} \mathrm{~F}$-Fluorodeoxyglucose (FDG) PET-CT showed ${ }^{18}$ F-FDG uptake (fig. 2a) in the sixth segment of the right lobe (standard uptake value, SUV, 4.6) and also at the liver hilum (SUV 3.1.).

The patient was enrolled in a phase II study (EudraCT No. 2005-005732-27) with SUN (50 mg/die) on a standard 4-week-on/2-week-off regimen. After the first cycle, a 25\% dose reduction was required because of grade III thrombocytopenia. The other reported toxicities were grade II fatigue, mild 
hypertension, grade I nausea and a cutaneous rash. The PET-CT scan performed after the first cycle showed metabolic stabilization of the disease according to EORTC criteria [8], with a reduction in SUV $<15 \%$ (4.1 vs. 4.6 at the sixth segment and 2.8 vs. 3.1 at the liver hilum). A CT scan performed after the second cycle confirmed a stable disease.

Ten days after the start of the third cycle, the patient experienced severe loss of appetite and nausea, followed, in a few hours, by copious hematemesis and hemorrhagic shock. Emergency gastroscopy revealed hemorrhagic gastritis. Prompt treatment with plasma substitutes, blood transfusions, vasoactive drugs and antibiotics was started, and SUN was discontinued.

The patient recovered and his clinical status constantly improved in the following weeks without any further treatment. Twelve months after the hematemesis (April 2009) episode, a PET-CT scan did not indicate any sign of the tumor (complete response according to EORTC criteria; fig. 2b). In a CT scan performed at the same time, the left branch of the portal vein was completely canalized but enlarged, whereas the main trunk and the right branch were substituted by fibrotic, cord-like tissue, and collateral vessels were present at the liver hilum. No signs of HCC were detectable in the liver parenchyma (fig. 1b1-b3). The Child-Pugh score was A5 and the MELD score 9.

\section{Conclusions}

The introduction of SOR in the treatment of advanced HCC has resulted in great enthusiasm since it was the first drug to beneficially affect the survival rate in this setting, although some issues have been raised concerning cost-effectiveness and patient selection. However, the possibility of increasing survival even for $<3$ months may be considered the beginning of a new era in the treatment of HCC, and it has given incentive to evaluate other agents and to target alternative pathways.

SUN is a multikinase inhibitor, but it shares the mechanism of action of SOR only partially, because of its stronger inhibition of VEGFR-related pathways due to an interaction both with VEGFR1 and VEGFR2 [9]. In the case reported here, a complete metabolic response and a partial morphologic response were achieved, lasting $>1$ year despite the interruption of SUN at the beginning of the third cycle. This excellent outcome was accompanied by re-canalization of PVTT and liver function improvement. The response seemed predicted by SUV reduction on the PET-CT scan. Although the sensitivity of ${ }^{18} \mathrm{~F}$-FDG-PET for HCC is about $50 \%$ and it is well known that ${ }^{18} \mathrm{~F}$-FDG uptake is associated with tumor differentiation $[10],{ }^{18} \mathrm{~F}-\mathrm{FDG}$ uptake is significantly higher in poorly differentiated HCC and may be a good predictor of outcome in these patients [11].

A clinical response according to EORTC criteria and RECIST is very rare in HCC. In the SHARP study [6], only 2 partial responses were observed in 299 patients treated with SOR. However, it must be considered that in our patient treatment was complicated by a potentially life-threatening side effect which required hospitalization in an intensive care unit. Even if gastrointestinal bleeding is a very common adverse event in PVTT patients, we cannot exclude a relationship between treatment and the complication due to the interaction between SUN and the coagulation pathway. This case clearly stresses the narrow therapeutic window of multikinase inhibitors in HCC originating from liver cirrhosis. The same mechanism of action that interferes with tumor growth might affect the liver vasculature already impaired by cirrhosis. The brief exposure to SUN needed in our patient to obtain both tumor response and gastrointestinal bleeding suggests further studies on the effectiveness of SUN in this setting as well as on the duration of treatment with multikinase inhibitors in HCC. 


\begin{tabular}{|c|c|c|c|}
\hline $\begin{array}{c}\text { Case Reports in } \\
\text { O.uit: }\end{array}$ & $\begin{array}{l}\text { Case Rep Oncol 2010;3:391-396 } \\
\text { DOI: } 10.1159 / 000322135\end{array}$ & $\begin{array}{l}\text { Published online: } \\
\text { November 6, } 2010\end{array}$ & $\begin{array}{l}\text { O } 2010 \text { S. Karger AG, Basel } \\
\text { ISSN } 1662-6575 \\
\text { www.karger.com/cro }\end{array}$ \\
\hline
\end{tabular}

Although SUN treatment in advanced HCC has been evaluated only in a few phase-II studies and resulted in poor outcome $[12,13]$, our case report may attract further interest in this drug, since the response rate with SOR is very low despite its survival benefit; moreover, no specific evaluation of patients with PVTT was made. Targeting a different set of tyrosine kinase receptors, including VEGFR1 and VEGFR2, PDGFR and c-KIT, SUN, or other multikinase inhibitors, might exert a different effect both on cancer cells and the coagulation pathway compared to SOR, which mainly interferes with the Raf/MEK/ERK pathway [14].

In conclusion, in this patient with advanced HCC, SUN was highly effective not only in terms of metabolic and morphologic responses, but also of PVTT recanalization. As far as we know, this effect has never been reported using this tyrosine kinase inhibitor.

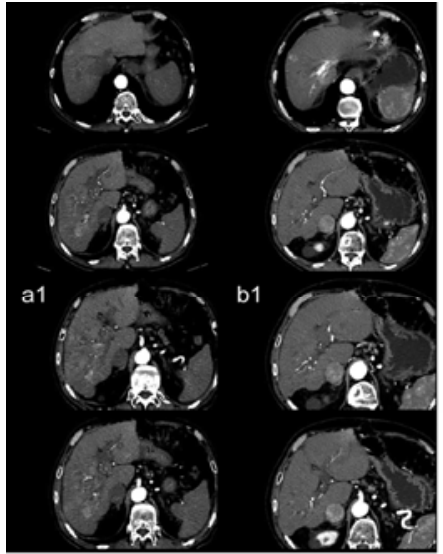

early arterial phase

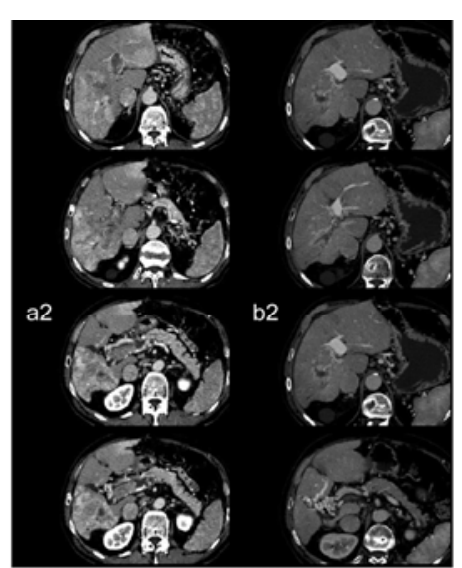

late arterial phase

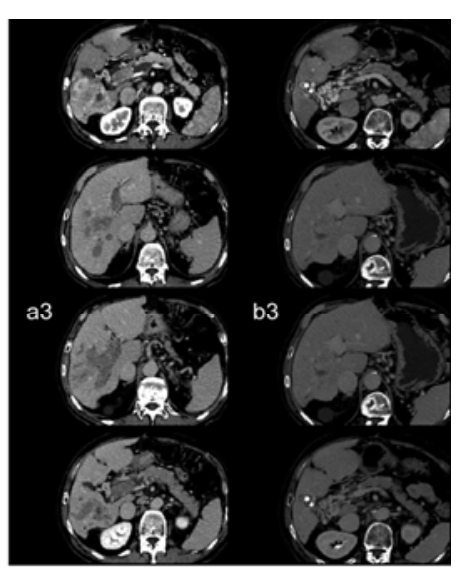

portal phase

Fig. 1. CT scan at baseline (a1-a3) and 1 year later (b1-b3). 


\begin{tabular}{|c|c|c|c|}
\hline $\begin{array}{c}\text { Case Reports in } \\
\text { Cicis: }\end{array}$ & \begin{tabular}{|l} 
Case Rep Oncol 2010;3:391-396 \\
DOI: 10.1159/000322135
\end{tabular} & $\begin{array}{l}\text { Published online: } \\
\text { November 6, } 2010\end{array}$ & $\begin{array}{l}\text { O } 2010 \text { S. Karger AG, Basel } \\
\text { ISSN 1662-6575 } \\
\text { www.karger.com/cro }\end{array}$ \\
\hline
\end{tabular}
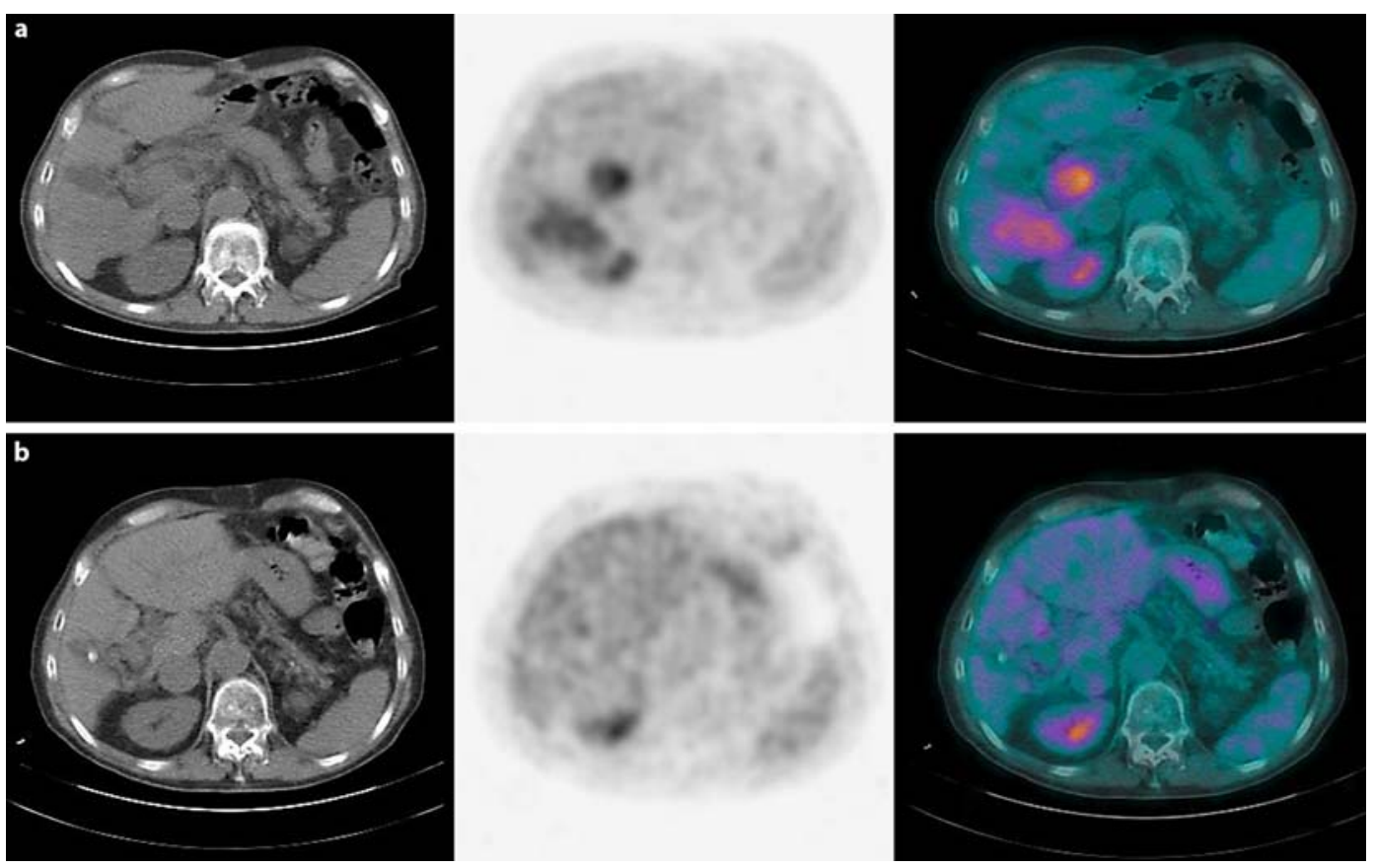

Fig. 2. PET-CT scan at baseline (a) and 1 year later $(\mathbf{b})$.

\section{References}

1 Stuart KE, Anand AJ, Jenkins RL, et al: Hepatocellular carcinoma in the United States. Prognostic features, treatment outcome and survival. Cancer 1996;77:2217-2222.

$\checkmark 2$ Calvet X, Bruix J, Gines P, et al: Prognostic factors of hepatocellular carcinoma in the west: a multivariate analysis in 206 patients. Hepatology 1990;12:753-760.

-3 Llovet JM, Bustamante J, Castells A, et al: Natural history of untreated nonsurgical hepatocellular carcinoma: rationale for the design and evaluation of therapeutic trials. Hepatology 1999;29:62-67.

4 Yamada R, Sato M, Kawabata M, et al: Hepatic artery embolization in 120 patients with unresectable hepatoma. Radiology 1983;148:397-401.

5 Minagawa M, Makuuchi M, Takayama T, et al: Selection criteria for hepatectomy in patients with hepatocellular carcinoma and portal vein tumor thrombus. Ann Surg 2001;233:379-384.

6 Llovet JM, Ricci S, Mazzaferro V, et al: Sorafenib in advanced hepatocellular carcinoma. N Eng J Med 2008;359:378-390.

7 Elice F, Rodeghiero F, Falanga A, Rickles FR: Thrombosis associated with angiogenesis inhibitors. Best Pract Res Clin Haematol 2009;22:115-128.

-8 Young H, Baum R, Cremerius U, et al: Measurement of clinical and subclinical tumour response using $\left[{ }^{18} \mathrm{~F}\right]$ fluorodeoxyglucose and positron emission tomography: review and 1999 EORTC recommendations European Organization for Research and Treatment of Cancer (EORTC) PET Study Group. Eur J Cancer 1999;35:1773-1782.

9 Mendel DB, Laird AD, Xin X, et al: In vivo antitumor activity of SU11248, a novel tyrosine kinase inhibitor targeting vascular endothelial growth factor and platelet-derived growth factors receptors: determination of a pharmacokinetic/pharmacodynamic relationship. Clin Cancer Res 2003;9:327-337.

10 He YX, Guo QY: Clinical applications and advances of positron emission tomography with fluorine 18flurodeoxyglucose (18F-FDG) in the diagnosis of liver neoplasms. Postgrad Med J 2008;84:246-251.

-11 Seo S, Hatano E, Higashi T, Hara T, Tada M, et al: Fluorine 18-Fluorodeoxyglucose positron emission tomography predicts tumor differentiation, P-glycorotein expression, and outcome after resection in hepatocelular carcinoma. Clin Cancer Res 2007;13:427-433. 
12 Faivre SJ, Raymond E, Boucher E, et al: Safety and efficacy of sunitinib in patients with advanced hepatocellular carcinoma: an open label, multicentre, phase II study. Lancet Oncol 2009;10:794-800.

13 Zhu AX, Sahani DV, di Tommaso E, et al: A phase II study of sunitinib in patients with advanced hepatocellular carcinoma. J Clin Oncol 2009;24:1896-1903.

14 Gollob JA, Wilhelm S, Carter C, et al: Role of Raf kinase in cancer: therapeutic potential of targeting the Raf/MEK/ERK signal transduction pathway. Semin Oncol 2006;33:392-406. 